\title{
Safety and Tolerability
}

National Cancer Institute

\section{Source}

National Cancer Institute. Safety and Tolerability. NCI Thesaurus. Code C142676.

The aspects of medical product evaluation associated with risk to the subject/patient and the degree to which overt adverse effects can be tolerated. 